\title{
EXISTENCE OF SOLUTIONS FOR THE ANTI-PLANE STRESS FOR A NEW CLASS OF "STRAIN-LIMITING" ELASTIC BODIES
}

\author{
MIROSLAV BULÍČEK, JOSEF MÁLEK, K. R. RAJAGOPAL, AND JAY R. WALTON
}

\begin{abstract}
The main purpose of this study is to establish the existence of a weak solution to the anti-plane stress problem for a class of recently proposed new models that could describe elastic materials in which the stress can increase unboundedly while the strain yet remains small. We shall also investigate the qualitative properties of the solution that is established. Although the equations governing the deformation that are being considered share certain similarities with the minimal surface problem the presence of an additional model parameter that appears in the equation and its specific range makes the problem, as well as the result, different from those associated with the minimal surface problem.
\end{abstract}

\section{INTRODUCTION}

Few models within the context of continuum mechanics have had the success of the linearized elastic solid model. Its great success notwithstanding, there are classes of problems to which the solutions provided by the linearized theory of elasticity is far from satisfactory, namely in the prediction of the strains and stresses at and near the tip of a crack and the propagation of cracks. The problem of fracture engaged the attention of Galileo [14] who in fact states that the problem had attracted attention much before his studies into fracture began ${ }^{1}$. Ever since then the problem of fracture has held the attention of physicists, engineers and applied mathematicians, but despite all this interest important open issues remain unresolved. The problem with using linearized elasticity to study the problems of stresses and strains around cracks stems from the fact that the relationship between the stress and the strain is linear and thus as the stress increases and becomes unbounded, the strain also increases and becomes unbounded, thereby violating the starting assumption in the linearization procedure that the strains are sufficiently small so that higher order terms in the strain can be neglected. In order to avoid the singularity that arises in the strains, a variety of ad hoc approaches ${ }^{2}$ have been used

2000 Mathematics Subject Classification. 35Q74,74B20,49Q05.

Key words and phrases. Plane stress, V-notch, Linearized strain, weak solution, minimal surface.

M. Bulíček and J. Málek acknowledge the support of the project LL1202 in the programme ERC-CZ funded by the Ministry of Education, Youth and Sports of the Czech Republic. K. R. Rajagopal thanks the National Science Foundation for its support of his work. He and J. R. Walton acknowledge support by Award No. KUS-C1-016-04 from King Abdullah University of Science and Technology.

${ }^{1}$ Galileo refers to Aristotle's interest in the problem.

${ }^{2}$ There have been additional approaches to modeling fracture by introducing additional forces and additional balance laws. Unfortunately, many of the quantities introduced in such theories 
(see Broberg [7], C herepanov [9], Kaninnen and Popelar [17] for details). These approaches vary based on whether the material in question is ductile or brittle. One such approach that is applied to bodies that are expected to become ductile in a neighborhood of the crack tip where the stresses are high, is the introduction of crack surface cohesive zones and a process zone at the crack tip (see Broberg [7]) or plasticity in the neighborhood of the crack tip (see Broberg [7], Kaninnen and Popelar [17]). In the case of brittle fracture, which is relevant to geomaterials such as rocks and also to metals at sufficiently low temperatures, there is no significant inelastic response near the crack tip and one uses either the maximum tensile stress or the Mohr-Coulomb criterion which is based on the shear stress on a plane reaching a critical value that depends on the normal stress on the plane (see Nadai [25]), to determine crack propagation. Several modi fications have been made to the MohrCoulomb criterion to study the fracture of brittle materials but we shall not discuss them here.

There have been several attempts to describe fracture from the atomistic level and bootstrapping the same to the continuum level. Popular amongst such numerical approaches are those appealing to the Monte Carlo method and those that employ the finite element method. These approaches which are mainly computational in nature involve adjustable parameters that come into play in each situation. The exact physical relevance of these parameters is not completely clear. Contrary to the claims to the effect that they are more fundamental with less approximations being made, the results depend critically on assumptions at a more fundamental level concerning the force potentials and the nature of the interactions between the atoms. The point that needs to be recognized is that the modeling is at the atomistic level and even a small error gets magnified due to the numerous iterations that are carried out.

Recently, there have been several papers (see Kim, Schiavone and Ru [18], [20], [21], Kim, Ru and Schiavone [19], Antipov and Schiavone [1]) that study fracture in brittle materials appealing to an approach by Murdoch and Gurtin [16] that introduces surface elasticity at the surface of cracks. These studies modify the classical linearized elasticity theory by allowing for elastic surface energy. The authors claim that the solutions that they obtain for the problem lead to bounded stresses and strains. Unfortunately, in all these papers, the authors make the same mistake and as pointed out by Walton [49] the solutions that they obtain do not predict bounded strains and stresses - these quantities are singular, the singularity being logarithmic. This thus leaves open the question of generalizing the classical theory, while yet retaining the notion of the linearized strain, to lead to a theory that predicts bounded stra ins and arbitrary stresses at a crack tip.

With a view towards providing a sound thermodynamic basis to fracture within the context of a theory that appeals to linearized strain, Sendova and Walton [40] have recently developed a procedure that would be applicable to brittle materials endowed with cracks. This work builds on the earlier studies of Slattery [41] and Oh et al. [30] by introducing long range interaction forces in the vicinity of the crack tip. Sendova and Walton [40] treat the crack surface as a dividing surface and endow

have never been measured and the need to introduce such additional quantities and an additional balance law are far from compelling. As no specific boundary value problem has been solved within the context of such recondite theories one cannot even evaluate the usefulness of such theories even from the point of view of merely carrying out a parametric study. 
the surface with a surface energy. They also introduce several additional properties associated with the surface such as mass, momentum, stress, and entropy. The theory predicts a finite stress at the crack tip and an alternative fracture criterion which states that the crack will propagate if the stress at the crack tip is equal to or greater than a particular value. Such a fracture criterion is easy to understand and seems physically reasonable. However, the theory is a non-local theory that removes the singularities that are present in the classical theory, and is at the cost of a very complicated formulation for specific boundary value problems, leading to very daunting computational problems.

It is natural to enquire whether within the context of non-linear elastic models the singularities in the stresses and strains disappear. In a series of papers concerning both compressible and incompressible non-linear elastic bodies, Knowles and Sternberg (see [44], [45], [46], [47]) and Knowles [22]) have carried out an asymptotic analysis of the stresses and strains in the vicinity of a crack tip for special non-linear elastic bodies, for plane strain and anti-plane strain problems. Their studies unfortunately do not lead to bounded solutions for the strains near a crack tip thereby leaving open the question whether bounded strains at crack tips are possible. With regard to elastic solids capable of large deformations, this question was answered in the affirmative for a special class of non-linear elastic materials by Tarantino [48]. He showed that the singularities in the stresses disappear and the stresses remain bounded in plane stress problems at a crack tip in the case of a material which obeys the Bell constraint (see Bell [5], [6]) ${ }^{3}$. Based on his experimental results, Bell proposed the constraint that the $\operatorname{tr} \mathbf{V}=3$, where $\mathbf{V}$ is the left Cauchy stretch tensor. The bodies under consideration undergo large deformations in the inelastic range. Unfortunately, a material that obeys the Bell constraint cannot support simple shear undermining the efficacy of the class of materials being used to describe the response of real bodies. Also, it seems that the constraint really concerns the inelastic response of solids wherein such a constraint maybe reasonable.

As mentioned earlier, for brittle materials, fracture seems to occur at small strains with negligible inelastic response. Thus, one is faced with the following question: for brittle materials is it possible to develop a small strain theory with the response being essentially elastic? Recently, Rajagopal and Walton [39] have answered the question in the affirmative for the problem of a crack subject to anti-plane strain. However, in order to answer the question in the affirmative, they have to go beyond the realms of the classical linearized theory of elasticity or for that matter the general theory of Cauchy elasticity and appeal to a novel generalization to describe the response of elastic bodies. The generalization of the class of elastic bodies that they appeal to belongs to the class of elastic bodies introduced by Rajagopal (see Rajagopal [31]). Instead of restricting oneself to the class of the classical Cauchy elastic or Green bodies ${ }^{4}$, Rajagopal [32], [35], [34] suggested the possibility of implicit models for elastic bodies that greatly enhances the class of elastic bodies. A rigorous thermodynamic basis has also been provided for such elastic bodies by Rajagopal and Srinivasa [36], [37] and Rajagopal and Tao

\footnotetext{
${ }^{3}$ Beatty and Hayes have studied an elastic material that obeys the Bell constraint in great detail (see [2], [3], [4]).

${ }^{4}$ The variou s interpretations of what was considered as an elastic material has been discussed in a review article titled "Conspectus of concepts of elasticity" by Rajagopal [33].
} 
[38]. Interestingly, when such implicit constitutive relations are linearized using the standard linearization procedure, namely that the trace norm of the displacement gradient is of order $O(\delta), \delta<<1$, it is possible to obtain a sub-class of constitutive relations wherein the linearized strain is a non-linear function of the stress, see [35]. Moreover, it is possible to obtain such constitutive relations which lead to limiting values for the linearized stra in. Rajagopal and Walton [39] working within the framework of such a limiting strain theory and using the methods of asymptotic analysis (perturbation method) were able to show that unlike the result in the case of the classical linearized solid, the stresses and the strains remain bounded as one approaches the crack tip, and one finds that the opposite faces of the crack undergo a displacement that is shaped like a cusp. More recently, Kulvait et al. [23] studied the anti-plane stress problem numerically by using the finite element method. They find that their numerical solutions are stable and provide some information regarding the nature of the solution near the tip of a V-notch. In particular, they observe stress concentration in the vicinity of the singularity.

In this paper, we study the question of existence of solution to an elastic body that is characterized by a constitutive relation of the type considered by Rajagopal and Walton [39] and Kulvait et al. [23], subject to a state of anti-plane stress. This reduces the problem to a scalar equation that shares certain similarities with the Euler-Lagrange equation for the minimal surface problem. In fact, the model that is considered contains an additional parameter and for a particular value of the parameter the model coincides with the minimal surface optimality equation. We investigate the problem for a range of model parameters so that the results that are presented are different (yet close) with those associated with the minimal surface problem.

The organization of the paper is as follows. In the next section we introduce the model, derive the governing equation for the anti-plane stress problem, define its weak solution, and finally formulate the main result concerning its existence and the properties of the solution. In section 3 we introduce an $\delta$-approximate problem that moves the setting of the original problem from $W^{1,1}(\Omega)$ to a problem in $W^{1,1+\delta}(\Omega)$; for fixed $\delta$ the existence of weak solution to the $\delta$-approximate problem is standard and it is known that the solution is smooth for smooth data. In Section 4 , we focus on the derivation of the estimates up to the boundary that are uniform with respect to $\delta$. These estimates are achieved using techniques of regularity theory; near the boundary we apply the method of barrier sub- and super-solutions performed on the convex parts of the boundary and the possibility to extend the solution from the interior to th e exterior on the remaining flat parts of the boundary. These estimates are sufficient for taking the limit, as $\delta \rightarrow 0+$, from $\delta$-approximate problems to the solution of the original problem. The last step in the proof of the main result is presented in the final Section 5.

\section{The Formulation of The PROBlem AND THE MAIN RESUlt}

2.1. General formulation of the problem. We suppose that an elastic body that has undergone a deformation such that the displacement gradient (and hence the strain) is small, is in equilibrium and occupies a configuration in a three dimensional space and is denoted by the set $B$ with the boundary $\partial B$. In addition, we suppose that the body is in equilibrium due to the loading on its boundary. More specifically, we consider the problem of determining the displacement $\boldsymbol{u}: B \rightarrow \mathbb{R}^{3}$, 
the Cauchy stress $\boldsymbol{T}: B \rightarrow \mathbb{R}_{\text {sym }}^{3 \times 3}$ and the linearized strain $\varepsilon: B \rightarrow \mathbb{R}_{\text {sym }}^{3 \times 3}$ so that

$$
\begin{aligned}
\operatorname{div} \boldsymbol{T} & =0, \quad \boldsymbol{G}(\boldsymbol{T}, \boldsymbol{\varepsilon})=\mathbf{0} \text { and } \quad \boldsymbol{\varepsilon}=\frac{\nabla \boldsymbol{u}+(\nabla \boldsymbol{u})^{T}}{2} \quad \text { in } B \\
\boldsymbol{T} \boldsymbol{\nu} & =\boldsymbol{g} \quad \text { on } \partial B
\end{aligned}
$$

where $\boldsymbol{G}: \mathbb{R}_{\text {sym }}^{3 \times 3} \times \mathbb{R}_{\text {sym }}^{3 \times 3} \rightarrow \mathbb{R}_{\text {sym }}^{3 \times 3}$ is supposed to be of the form

$$
\boldsymbol{G}(\boldsymbol{T}, \boldsymbol{\varepsilon})=\boldsymbol{\varepsilon}-\Phi\left(\operatorname{tr} \boldsymbol{T}, \operatorname{tr} \boldsymbol{T}^{2}\right) \boldsymbol{I}-\Psi\left(\operatorname{tr} \boldsymbol{T}^{2}\right) \boldsymbol{T} \text { with } \Phi(0, .)=0,
$$

and $\boldsymbol{\nu}: \partial \Omega \rightarrow \mathbb{R}^{3}$ is the outer normal.

The first equation in (1) represents the equilibrium equation (the balance of linear momentum wherein the inertial effects and the influence of the external body forces are neglected) and the third equation of (1) links the displacement field to the linearized strain tensor $\varepsilon$. In simply connected open sets the existence of $\boldsymbol{u}$ fulfilling $(1)_{3}$ to a (given) $\varepsilon$ is equivalent to the tensorial equation

$$
\text { curl curl } \varepsilon=0 \text { in } B \text {, }
$$

which is usually referred to as the compatibility condition. Note that such $\boldsymbol{u}$ is determined uniquely modulo the field $\boldsymbol{a}+\boldsymbol{b} \times x$, where $\boldsymbol{a}$ and $\boldsymbol{b}$ are arbitrary vectors.

Regarding boundary conditions, we restrict ourselves to fixing the traction over all the boundary $\partial \Omega$. (This restriction is due to our considering the anti-plane stress problem below in this work.)

As pointed out recently by Rajagopal [32], the framework specified by the implicit constitutive equation $\boldsymbol{G}(\boldsymbol{T}, \boldsymbol{\varepsilon})=\mathbf{0}$ tremendously enlarges the class of models in the elasticity theory wherein one is concerned with the linearized strain and these models are then capable of describing a non-linear relationship between the strains and stresses (which could be arbitrarily large), even though the strains are small. One class of such models has been proposed by Rajagopal (see [35], [8]), where

$$
\boldsymbol{\varepsilon}=\beta\left(1-\exp \frac{-\lambda \operatorname{tr} \boldsymbol{T}}{\left(1+|\boldsymbol{T}|^{b}\right)^{1 / b}}\right) \boldsymbol{I}+\frac{\boldsymbol{T}}{2 \mu\left(1+\kappa|\boldsymbol{T}|^{a}\right)^{1 / a}},
$$

where $|\boldsymbol{T}|:=\left(\operatorname{tr} \boldsymbol{T}^{2}\right)^{1 / 2}=(\boldsymbol{T}: \boldsymbol{T})^{1 / 2}=\left(\sum_{i, j=1,2,3} T_{i j}^{2}\right)^{\frac{1}{2}}$ and $a, b, \beta, \lambda, \mu$ and $\kappa$ are positive material constants.

The limiting strain model (5) has been designed to describe the response of materials in which the stress can be high while the strains yet remain small.

The models exhibiting such characteristics may be for example used to describe the behavior of brittle materials near the tips of cracks or notches, see Kulvait et. al. [23] or Rajagopal and Walton [39] for further details.

In this study focused on the analysis of the problem (1)-(2) we consider (3) with $\Psi$ of the form

$$
\Psi\left(|\boldsymbol{T}|^{2}\right)=\frac{1}{\left(1+|\boldsymbol{T}|^{a}\right)^{1 / a}} \text { with } a>0 .
$$

Note that up to the constants (that do not play any role in the analysis presented below), (5) belongs to the class that is being considered. The form of $\Phi$ in (3) is not important here as we restrict ourselves to the anti-plane stress problem that we shall describe next. 
2.2. Anti-plane stress problem. In this study we reduce the complexity of the problem (1)-(3), (6) by restricting ourselves to bodies that are in a state of antiplane stress. Anti-plane stress is a problem as sketched in Fig. 1, where

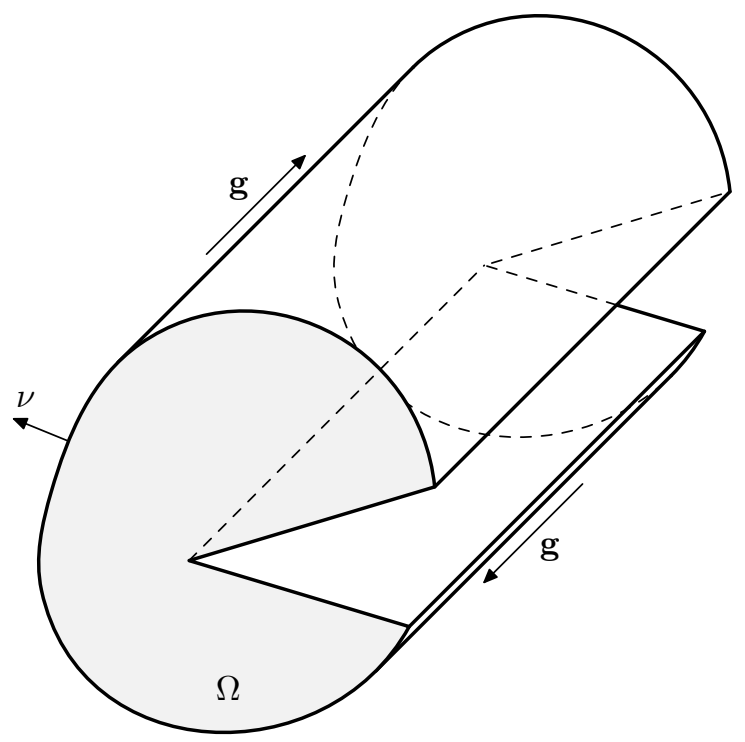

Figure 1. Anti-plane stress geometry.

$$
\begin{aligned}
& B=\Omega \times \mathbb{R} \text { and } \Omega \subset \mathbb{R}^{2} \text { is a simply connected open set, } \\
& \boldsymbol{u}=\boldsymbol{u}\left(x_{1}, x_{2}\right)=\left(0,0, u\left(x_{1}, x_{2}\right)\right), \quad \boldsymbol{g}=(0,0, g),
\end{aligned}
$$

and

$$
\boldsymbol{T}=\left(\begin{array}{ccc}
0 & 0 & T_{13}\left(x_{1}, x_{2}\right) \\
0 & 0 & T_{23}\left(x_{1}, x_{2}\right) \\
T_{13}\left(x_{1}, x_{2}\right) & T_{23}\left(x_{1}, x_{2}\right) & 0
\end{array}\right)
$$

Under such circumstances, it then follows from (3) that the only nonzero components of the linearized strain are $\varepsilon_{13}$ and $\varepsilon_{23}$ and they also depend on $x_{1}$ and $x_{2}$.

The problem under consideration (1)-(3) then simplifies to

$$
\begin{aligned}
-\frac{\partial T_{13}}{\partial x_{1}}-\frac{\partial T_{23}}{\partial x_{2}}=0 & \text { in } \Omega, \\
\varepsilon_{13}=\Psi\left(|\boldsymbol{T}|^{2}\right) T_{13} \text { and } \varepsilon_{23}=\Psi\left(|\boldsymbol{T}|^{2}\right) T_{23} & \text { in } \Omega, \\
\varepsilon_{13}=\frac{1}{2} \frac{\partial u}{\partial x_{1}} \text { and } \varepsilon_{23}=\frac{1}{2} \frac{\partial u}{\partial x_{2}} & \text { in } \Omega, \\
T_{13} \nu_{1}+T_{23} \nu_{2}=g & \text { on } \partial \Omega .
\end{aligned}
$$

While originally we had the system of 15 equations for the components of the symmetric tensors $\boldsymbol{T}$ and $\varepsilon$ and the displacement $\boldsymbol{u}$, the reduced system consists of five (in fact three) equations for five scalars $u, T_{13}, T_{23}, \varepsilon_{13}, \varepsilon_{23}$ (in fact $u, T_{13}$, $\left.T_{13}\right)$. 
In simply connected domains the existence of $u$ for given $\varepsilon_{13}$ and $\varepsilon_{23}$ fulfilling (12) is equivalent to the compatibility condition

$$
\frac{\partial \varepsilon_{23}}{\partial x_{1}}-\frac{\partial \varepsilon_{13}}{\partial x_{2}}=0 \quad \text { in } \Omega
$$

The setting given by (10)-(13), (14) can be simplified further by introducing the Airy stress function $U: \Omega \rightarrow \mathbb{R}$ satisfying

$$
T_{13}=\frac{1}{\sqrt{2}} \frac{\partial U}{\partial x_{2}} \quad \text { and } \quad T_{23}=-\frac{1}{\sqrt{2}} \frac{\partial U}{\partial x_{1}} .
$$

Then the equilibrium equation (10) is automatically met and (14) together with (11) and (13) leads to

$$
\begin{array}{r}
\frac{\partial}{\partial x_{2}}\left(\Psi\left(|\nabla U|^{2}\right) \frac{\partial U}{\partial x_{2}}\right)+\frac{\partial}{\partial x_{1}}\left(\Psi\left(|\nabla U|^{2}\right) \frac{\partial U}{\partial x_{1}}\right)=0 \quad \text { in } \Omega \\
\frac{\partial U}{\partial x_{2}} \nu_{1}-\frac{\partial U}{\partial x_{1}} \nu_{2}=\sqrt{2} g \quad \text { on } \partial \Omega .
\end{array}
$$

Let us assume further, merely for simplicity, that the boundary $\partial \Omega$ can be parameterized by a closed curve $\gamma:[a, b] \rightarrow \mathbb{R}^{2}\left(\gamma(s)=\left(\gamma_{1}(s), \gamma_{2}(s)\right)\right), \gamma(a)=$ $\gamma(b)$ that is oriented counterclockwise. Then for $x=\left(\gamma_{1}(s), \gamma_{2}(s)\right) \in \partial \Omega$ the tangent vector $\boldsymbol{t}(x)=\frac{1}{\sqrt{\left(\gamma_{1}^{\prime}(s)\right)^{2}+\left(\gamma_{2}^{\prime}(s)\right)^{2}}}\left(\gamma_{1}^{\prime}(s), \gamma_{2}^{\prime}(s)\right)$, the outer normal $\boldsymbol{\nu}(x)=$ $\frac{1}{\sqrt{\left(\gamma_{1}^{\prime}(s)\right)^{2}+\left(\gamma_{2}^{\prime}(s)\right)^{2}}}\left(\gamma_{2}^{\prime}(s),-\gamma_{1}^{\prime}(s)\right)$ and $(17)$ yields

$$
\begin{aligned}
\sqrt{2} g(\gamma(s)) \sqrt{\left(\gamma_{1}^{\prime}(s)\right)^{2}+\left(\gamma_{2}^{\prime}(s)\right)^{2}} & =\frac{\partial U(\gamma(s))}{\partial x_{2}} \gamma_{2}^{\prime}(s)+\frac{\partial U(\gamma(s))}{\partial x_{1}} \gamma_{1}^{\prime}(s) \\
& =\frac{d}{d s} U(\gamma(s)) .
\end{aligned}
$$

Hence (17) leads to ( $x_{0} \in \partial \Omega$ fixed, $a_{0} \in \mathbb{R}$ arbitrary)

$$
U(x)=a_{0}+\sqrt{2} \int_{x_{0}}^{x} g(\gamma(s)) \sqrt{\left(\gamma_{1}^{\prime}(s)\right)^{2}+\left(\gamma_{2}^{\prime}(s)\right)^{2}} d s=: U_{0}(x) \quad \text { for } \quad x \in \partial \Omega .
$$

For simplicity we set $a_{0}=0$ in the sequel.

We conclude this subsection by summarizing the problem that we aim to solve and by stating the main result. Introducing for $i=1,2$ the notation $D_{i}:=\frac{\partial}{\partial x_{i}}$ and recalling the definition of $\Psi$ given in (6) we say that $U: \Omega \rightarrow \mathbb{R}$ solves Problem $\mathcal{P}$ if

$$
\begin{aligned}
-D_{i}\left(\frac{D_{i} U}{\left(1+|\nabla U|^{a}\right)^{\frac{1}{a}}}\right) & =0 & & \text { in } \Omega, \\
U & =U_{0} & & \text { on } \partial \Omega,
\end{aligned}
$$

where we apply the summation convention in (20), and thus take the sum over the index $i$ from 1 to 2 . Note that the original Neumann-type problem (1)-(3) results in the non-homogeneous Dirichlet problem (20)-(21). 
In what follows we make the following assumptions concerning $\Omega$ and $U_{0}$ :

$\Omega \subset \mathbb{R}^{2}$ is a simply connected domain with $C^{0,1}$ boundary $\partial \Omega$ consisting of two subsets $\partial \Omega_{1} \cup \partial \Omega_{2}$ so that $\partial \Omega_{1}:=\{x \in \partial \Omega ; \boldsymbol{t}(x) \cap \Omega=\emptyset\}$.

Both $\partial \Omega_{1}$ and $\partial \Omega_{2}$ may consist of finitely many parts; each of them is parametrized by $\gamma=\gamma(s)$ restricted to an open interval. Each part of $\partial \Omega_{2}$ is flat and $U_{0}$ is constant there and on each uniformly convex part of $\partial \Omega_{1}, U_{0}$ is a $C^{1,1}$-function.

The main result of this study is stated in the following theorem.

Theorem 2.1. Let $\Omega \subset \mathbb{R}^{2}$ fulfill (22). Let further $\tilde{U}_{0} \in W^{1, \infty}(\Omega)$ be such that $\left.\tilde{U}_{0}\right|_{\partial \Omega}=U_{0}$ and suppose $U_{0}$ fulfills the conditions specified in $(22)$. Let $a \in(0,2)$. Then there is a unique weak solution $U \in W_{\text {loc }}^{2,2}(\Omega)$ to Problem $\mathcal{P}$ satisfying

$$
\begin{aligned}
& U-\tilde{U}_{0} \in W_{0}^{1,1}(\Omega), \\
& \left(\frac{\nabla U}{\left(1+|\nabla U|^{a}\right)^{\frac{1}{a}}}, \nabla \phi\right)=0 \quad \text { for all } \phi \in W_{0}^{1,1}(\Omega) .
\end{aligned}
$$

Let us first make a few remarks concerning the difficulty of the Problem $\mathcal{P}$ when considered in general in non-convex domains, see Fig. 1 or Fig. 2 below. Multiplying $(20)$ by $U-\tilde{U}_{0}$, where $\tilde{U}_{0}: \Omega \rightarrow \mathbb{R}$ is such that its trace on the boundary equals (almost everywhere if needed) to $U_{0}$, integrating the result over $\Omega$, then applying integration by parts and finally using some simple manipulations including the Hölder inequality we arrive at

$$
\|\nabla U\|_{1} \leq C(|\Omega|)\left\|\nabla \tilde{U}_{0}\right\|_{1} .
$$

This estimate places the problem close to the minimal surface problem in which the parameter $a=2$. It is well known that the space $W^{1,1}(\Omega)$ is not reflexive and the space of $B V$-functions or Radon measures has been used to study such problems. There are also counterexamples showing that solution for $a=2$ may not exist in general. More precisely, Finn in [13] and then also Nitsche in his survey paper [29] proved nonexistence of the classical solution for smooth data and nonconvex domains; later on the non-existence of the weak solution on nonconvex domains was established by Souček in [42]. For further references and for an overview of the state of the art to this topic we refer the interested reader to $[15,11,12]$.

The difficulty associated with such a setting consists in finding appropriate meaning to the value of such functions on the boundary. Here, we overcome this difficulty by incorporating regularity techniques that help us to observe that for smoother data the solution belongs to $W^{1,1}(\Omega) \cap W^{1, p}\left(\Omega \backslash K_{\varepsilon}\right)$, where $\varepsilon>0$ is arbitrary and $K_{\varepsilon}$ is an intersection of $\partial \Omega$ with the union of $\varepsilon$-balls centered in the corners of $\partial \Omega$. Since the nonlinear operator is monotone it is then easy to establish the existence of a (weak) solution provided that one has some suitable approximate problems at oneself's disposal. Here, we base the proof of Theorem (2.1) on solutions of the approximate problems $\mathcal{P}_{\delta}$ that are defined in the following subsection and that moves the setting of the problem from $W^{1,1}(\Omega)$ to the $W^{1,1+\delta}(\Omega)$ framework. Consequently, for fixed $\delta>0$ it is not difficult to establish the existence of a weak solution to the Problem $\mathcal{P}_{\delta}$ and to show that this solution is as smooth as needed and as the data permit. Then we derive the estimates up to the boundary that are uniform with respect to $\delta$. These estimates are achieved using techniques 
of regularity theory; near the uniformly convex parts of the boundary we apply the method of barrier sub- and super-solutions and on the remaining flat parts of the boundary, where $U_{0}$ is constant, we use the possibility of extending the solution from interior to the exterior and then applying the interior regularity results. These estimates are sufficient for taking the limit, as $\delta \rightarrow 0+$, from $\delta$-approximate problems to the solution of the original problem.

\section{The approximate Problem $\mathcal{P}_{\delta}$ and the properties of its solution}

For any (small) $\delta>0$ we consider the following approximate Problem $\mathcal{P}_{\delta}$ : to find $U^{\delta}: \Omega \rightarrow \mathbb{R}$ satisfying

$$
\begin{aligned}
-D_{i}\left(\frac{D_{i} U^{\delta}}{\left(1+\left|\nabla U^{\delta}\right|^{a}\right)^{\frac{1-\delta}{a}}}\right)=0 & \text { in } \Omega, \\
U^{\delta} & =U_{0} \quad \text { on } \partial \Omega .
\end{aligned}
$$

The presence of $\delta$ in (26)-(27) leads to stronger apriori estimates namely instead of (25) we have

$$
\left\|\nabla U^{\delta}\right\|_{1+\delta} \leq C(|\Omega|)\left\|\nabla \tilde{U}_{0}\right\|_{1+\delta} .
$$

In addition the nonlinear differential operator on the left hand side of (27) generates a strictly monotone operator on $W^{1+\delta}(\Omega)$. Thus it is easy to establish the following result.

Lemma 3.1. Let $\Omega \subset \mathbb{R}^{2}$ be a simply connected domain with $C^{0,1}$ boundary. Let further $\tilde{U}_{0} \in W^{1,1+\delta}(\Omega)$ and $a>0$. Then for any $\delta>0$ there is a unique weak solution $U^{\delta} \in W^{1,1+\delta}(\Omega)$ to Problem $\mathcal{P}_{\delta}$ satisfying

$$
\begin{aligned}
& U^{\delta}-\tilde{U}_{0} \in W_{0}^{1,1+\delta}(\Omega), \\
& \left(\frac{\nabla U^{\delta}}{\left(1+\left|\nabla U^{\delta}\right|^{a}\right)^{\frac{1-\delta}{a}}}, \nabla \phi\right)=0 \quad \text { for all } \phi \in W_{0}^{1,1+\delta}(\Omega) .
\end{aligned}
$$

In order to underline a remarkable difference between the problem $\mathcal{P}_{\delta}$ and the problem $\mathcal{P}$ we state the following monotone-type inequality

$$
\begin{aligned}
\left(\frac{\boldsymbol{x}}{\left(1+|\boldsymbol{x}|^{a}\right)^{\frac{1-\delta}{a}}}\right. & \left.-\frac{\boldsymbol{y}}{\left(1+|\boldsymbol{y}|^{a}\right)^{\frac{1-\delta}{a}}}\right) \cdot(\boldsymbol{x}-\boldsymbol{y}) \\
\geq & (1-\delta) \int_{0}^{1} \frac{|\boldsymbol{x}-\boldsymbol{y}|^{2}}{\left(1+\mid \boldsymbol{y}+s(\boldsymbol{x}-\boldsymbol{y})^{a}\right)^{\frac{1-\delta}{a}+1}} d s \\
& +\delta \int_{0}^{1} \frac{|\boldsymbol{x}-\boldsymbol{y}|^{2}}{\left(1+\mid \boldsymbol{y}+s(\boldsymbol{x}-\boldsymbol{y})^{a}\right)^{\frac{1-\delta}{a}}} d s
\end{aligned}
$$

valid for any $\delta \in[0,1]$ and $\boldsymbol{x}, \boldsymbol{y} \in \mathbb{R}^{d}$. Obviously, this inequality provides better information if $\delta>0$ and it is known that the additional degeneracy in the case $\delta=0$ destroys several approaches that are useful in the analysis of the problem with $\delta>0$.

Since the work of De Giorgi [10], Nash [26] and Lieberman [24], it is well known that the problem $\mathcal{P}_{\delta}$ admits a smooth solution for smooth data ${ }^{5}$. We present no

\footnotetext{
${ }^{5}$ See also the papers by Nečas [27], [28] and Stará [43] that can serve as the source for the complete proofs on which our proof is based
} 
more details concerning the regularity of solution to Problem $\mathcal{P}_{\delta}$ for $\delta>0$ fixed (on domains with smooth boundary) as we will apply regularity techniques in order to derive the uniform estimates for $U^{\delta}$ (on non-convex domains with corners). In particular, we will show in the next section that for any $\varepsilon>0$

$$
\sup _{\delta>0}\left\|\nabla U^{\delta}\right\|_{2, \Omega \backslash K_{\varepsilon}}<\infty,
$$

where $K_{\varepsilon}$ is the intersection of $\partial \Omega$ with the union of $\varepsilon$-balls centered at the corners of $\partial \Omega$.

\section{UNIFORM ESTIMATES}

In this section we write, for simplicity, $U$ instead of $U^{\delta}$. Since the uniquely specified weak solution to Problem $\mathcal{P}_{\delta}$ is smooth (and thus classical) we can work with (26) instead of (30). First, we recall the first uniform estimate (28), which is of the form

$$
\|\nabla U\|_{1+\delta} \leq C(|\Omega|)\left\|\nabla \tilde{U}_{0}\right\|_{1+\delta}
$$

Next, denoting $S:=\left\|\tilde{U}_{0}\right\|_{\infty}$, we set

$$
\phi:=(U \mp S)_{ \pm}
$$

in (30) (note that it has zero trace) to deduce that

$$
\|U\|_{\infty} \leq\left\|\tilde{U}_{0}\right\|_{\infty} \leq C
$$

Finally, we continue with further uniform estimates based on higher regularity techniques. For such a purpose, we first derive the identity (and also consequently the inequality) for the second derivatives of $U$. Thus, applying $D_{k}$ to (26) we obtain the identity ${ }^{6}$

$$
-D_{i}\left(\frac{D_{i k} U}{\left(1+|\nabla U|^{a}\right)^{\frac{1-\delta}{a}}}-(1-\delta) \frac{|\nabla U|^{a-1} D_{i} U D_{k}|\nabla U|}{\left(1+|\nabla U|^{a}\right)^{\frac{1-\delta}{a}+1}}\right)=0 \text { in } \Omega .
$$

Next, multiplying (35) by $D_{k} U$ and taking the sum over $k(k=1,2)$ we conclude that

$$
\begin{gathered}
-D_{i}\left(\frac{D_{i k} U D_{k} U}{\left(1+|\nabla U|^{a}\right)^{\frac{1-\delta}{a}}}-(1-\delta) \frac{D_{k} U|\nabla U|^{a-1} D_{i} U D_{k}|\nabla U|}{\left(1+|\nabla U|^{a}\right)^{\frac{1-\delta}{a}+1}}\right) \\
+\frac{\left|\nabla^{2} U\right|^{2}}{\left(1+|\nabla U|^{a}\right)^{\frac{1-\delta}{a}}}-(1-\delta) \frac{|\nabla U|^{a-1} D_{i} U D_{k}|\nabla U| D_{i k} U}{\left(1+|\nabla U|^{a}\right)^{\frac{1-\delta}{a}+1}}=0 .
\end{gathered}
$$

A simple algebraic manipulation then leads to

$$
\begin{array}{r}
-D_{i}\left(\frac{|\nabla U| D_{i}|\nabla U|}{\left(1+|\nabla U|^{a}\right)^{\frac{1-\delta}{a}}}-(1-\delta) \frac{D_{k} U|\nabla U|^{a-1} D_{i} U D_{k}|\nabla U|}{\left(1+|\nabla U|^{a}\right)^{\frac{1-\delta}{a}+1}}\right) \\
+\delta \frac{\left|\nabla^{2} U\right|^{2}}{\left(1+|\nabla U|^{a}\right)^{\frac{1-\delta}{a}}}+(1-\delta) \frac{\left|\nabla^{2} U\right|^{2}}{\left(1+|\nabla U|^{a}\right)^{\frac{1-\delta}{a}+1}} \leq 0 .
\end{array}
$$

Thus, defining

$$
w:=|\nabla U|
$$

${ }^{6}$ Here, we use the abbreviation $D_{i k}:=\frac{\partial^{2}}{\partial x_{j} \partial x_{i}}$. 
and multiplying (37) by arbitrary nonnegative nondecreasing Lipschitz $f(w)$, we deduce

$$
\begin{array}{r}
-D_{i}\left(\frac{w D_{i} w f(w)}{\left(1+w^{a}\right)^{\frac{1-\delta}{a}}}-(1-\delta) \frac{f(w) D_{k} U w^{a-1} D_{i} U D_{k} w}{\left(1+w^{a}\right)^{\frac{1-\delta}{a}+1}}\right) \\
+(\delta+(1-\delta)) \frac{w f^{\prime}(w)|\nabla w|^{2}}{\left(1+w^{a}\right)^{\frac{1-\delta}{a}}}-(1-\delta) \frac{f^{\prime}(w) D_{k} U w^{a-1} D_{i} U D_{k} w D_{i} w}{\left(1+w^{a}\right)^{\frac{1-\delta}{a}+1}} \\
+\delta \frac{\left|\nabla^{2} U\right|^{2}}{\left(1+w^{a}\right)^{\frac{1-\delta}{a}}} f(w)+(1-\delta) \frac{\left|\nabla^{2} U\right|^{2}}{\left(1+w^{a}\right)^{\frac{1-\delta}{a}}+1} f(w) \leq 0,
\end{array}
$$

which again by a simple algebraic manipulation and by neglecting two terms with coefficient $\delta$ leads to

$$
\begin{aligned}
& -D_{i}\left(\frac{w D_{i} w f(w)}{\left(1+w^{a}\right)^{\frac{1-\delta}{a}}}-(1-\delta) \frac{f(w) D_{k} U w^{a-1} D_{i} U D_{k} w}{\left(1+w^{a}\right)^{\frac{1-\delta}{a}+1}}\right) \\
& +(1-\delta) \frac{\left|\nabla^{2} U\right|^{2} f(w)+w f^{\prime}(w)|\nabla w|^{2}}{\left(1+w^{a}\right)^{\frac{1-\delta}{a}}+1} \leq 0 .
\end{aligned}
$$

Next, we focus on the second term of this inequality. Setting

$$
F_{1}(s):=\int_{0}^{s} \frac{f(t) t^{a-1}}{1+t^{a}} d t
$$

we observe that

$$
\begin{aligned}
& \frac{f(w) D_{k} U D_{i} U w^{a-1} D_{k} w}{\left(1+w^{a}\right)^{\frac{1-\delta}{a}+1}}=\frac{D_{k} U}{\left(1+w^{a}\right)^{\frac{1-\delta}{a}}} \frac{f(w) w^{a-1} D_{i} U D_{k} w}{\left(1+w^{a}\right)} \\
& =\frac{D_{k} U}{\left(1+w^{a}\right)^{\frac{1-\delta}{a}}} D_{i} U D_{k} F_{1}(w) \\
& =\frac{D_{k} U}{\left(1+w^{a}\right)^{\frac{1-\delta}{a}}} D_{k}\left(D_{i} U F_{1}(w)\right)-\frac{w D_{i} w}{\left(1+w^{a}\right)^{\frac{1-\delta}{a}}} F_{1}(w) \\
& =D_{k}\left(\frac{D_{k} U D_{i} U}{\left(1+w^{a}\right)^{\frac{1-\delta}{a}}} F_{1}(w)\right)-\frac{w D_{i} w}{\left(1+w^{a}\right)^{\frac{1-\delta}{a}}} F_{1}(w),
\end{aligned}
$$

where for the last identity we used (26). Thus, defining in what follows

$$
F_{2}(s):=\int_{0}^{s} \frac{\left(f(t)+(1-\delta) F_{1}(t)\right) t}{\left(1+t^{a}\right)^{\frac{1-\delta}{a}}} d t
$$

we can rewrite (39) in a more compact form

$$
\begin{aligned}
& -\triangle F_{2}(w)+(1-\delta) \operatorname{div} \operatorname{div}\left(\frac{\nabla U \otimes \nabla U}{\left(1+w^{a}\right)^{\frac{1-\delta}{a}}} F_{1}(w)\right) \\
& +(1-\delta) \frac{\left|\nabla^{2} U\right|^{2} f(w)+w f^{\prime}(w)|\nabla w|^{2}}{\left(1+w^{a}\right)^{\frac{1-\delta}{a}}+1} \leq 0,
\end{aligned}
$$

which will be finally used to get the desired estimates. 
4.1. Uniform interior estimates. Multiplying (42) by $\eta^{2 k}$, where $\eta$ is a smooth nonnegative cut off function (vanishing in the vicinity of the boundary) and integrating twice by parts we deduce the following inequality (where we already neglected the nonnegative term with $f^{\prime}$ )

$$
\begin{aligned}
(1-\delta) & \int_{\Omega} \frac{\left|\nabla^{2} U\right|^{2} f(w) \eta^{2 k}}{\left(1+w^{a}\right)^{\frac{1-\delta}{a}+1}} d x \\
& \leq \int_{\Omega} F_{2}(w) \triangle \eta^{2 k} d x-(1-\delta) \int_{\Omega} \frac{D_{k} U D_{i} U}{\left(1+w^{a}\right)^{\frac{1-\delta}{a}}} F_{1}(w) \cdot D_{i k} \eta^{2 k} d x
\end{aligned}
$$

Since it directly follows from (40) and (41) that $F_{2}(w) \leq C\left(1+w^{1+\delta} F_{1}(w)\right)$, we observe from (43) that

$$
(1-\delta) \int_{\Omega} \frac{\left|\nabla^{2} U\right|^{2} f(w) \eta^{2 k}}{\left(1+w^{a}\right)^{\frac{1-\delta}{a}+1}} d x \leq C(\eta, k)\left(1+\int_{\Omega} w^{1+\delta} F_{1}(w) \eta^{2 k-2} d x\right) .
$$

In what follows we assume that $\delta \leq \frac{1}{2}$ and recall the restriction $a \in(0,2)$. We would also like to emphasize that all estimates below heavily rely on the $L^{\infty}$ bound (34). Without such information we would need to restrict ourselves only to the case $a \in(0,1)$.

Setting $f(w):=w^{p}$ for arbitrary $p \geq 2$, we see from (40) that $F_{1}(w) \leq C w^{p}$. Consequently, on inserting it into (44) we obtain, after simple algebraic estimates, that

$$
\int_{\Omega} \frac{\left|\nabla^{2} U\right|^{2} w^{p} \eta^{2 k}}{\left(1+w^{a}\right)^{\frac{1-\delta}{a}+1}} d x \leq C(a, \eta, k, p)\left(1+\int_{\Omega} w^{1+p+\delta} \eta^{2 k-2} d x\right) .
$$

To estimate the right hand side, we apply interpolation between $W^{2,2}$ and $L^{\infty}$. Thus, using integration by parts, the uniform estimate (34) and the Hölder inequality we deduce that (recall that we assume $p \geq 2$ for simplicity)

$$
\begin{aligned}
& \int_{\Omega} w^{1+p+\delta} \eta^{2 k-2} d x=\int_{\Omega} \nabla U \cdot \nabla U|\nabla U|^{p-1+\delta} \eta^{2 k-2} d x \\
& =-\int_{\Omega} U\left(\triangle U|\nabla U|^{p-1+\delta} \eta^{2 k-2}+(p-1+\delta)|\nabla U|^{p-2+\delta} \nabla U \cdot \nabla|\nabla U| \eta^{2 k-2}\right) \\
& \quad-\int_{\Omega} U|\nabla U|^{p-1+\delta} \nabla U \cdot \nabla \eta^{2 k-2} d x \\
& \leq C\left(p, k, \tilde{U}_{0}\right) \int_{\Omega}\left|\nabla^{2} U\right| w^{p-1+\delta} \eta^{2 k-2}+w^{p+\delta} \eta^{2 k-3} d x .
\end{aligned}
$$

Next we focus on the first term on the right hand side. Using Young inequality, we directly obtain

$$
\begin{aligned}
\int_{\Omega} \mid & \nabla^{2} U \mid w^{p-1+\delta} \eta^{2 k-2} d x \\
& =\int_{\Omega}\left(\frac{\left|\nabla^{2} U\right|^{2} w^{p} \eta^{2 k}}{\left(1+w^{a}\right)^{\frac{1-\delta}{a}+1}}\right)^{\frac{1}{2}} w^{\frac{p-2+2 \delta}{2}}\left(1+w^{a}\right)^{\frac{1-\delta+a}{2 a}} \eta^{k-2} d x \\
& \leq \varepsilon \int_{\Omega} \frac{\left|\nabla^{2} U\right|^{2} w^{p} \eta^{2 k}}{\left(1+w^{a}\right)^{\frac{1-\delta}{a}+1}} d x+C(\varepsilon) \int_{\Omega} w^{p-2+2 \delta}\left(1+w^{a}\right)^{\frac{1-\delta+a}{a}} \eta^{2 k-4} d x \\
& \leq \varepsilon \int_{\Omega} \frac{\left|\nabla^{2} U\right|^{2} w^{p} \eta^{2 k}}{\left(1+w^{a}\right)^{\frac{1-\delta}{a}+1}} d x+C(\varepsilon) \int_{\Omega}\left(1+w^{p-1+a+\delta}\right) \eta^{2 k-4} d x
\end{aligned}
$$


Hence, on inserting this estimate into (46) we obtain (assuming that $k \geq 2$ )

$$
\begin{aligned}
\int_{\Omega} w^{1+p+\delta} \eta^{2 k-2} d x & \leq \varepsilon \int_{\Omega} \frac{\left|\nabla^{2} U\right|^{2} w^{p} \eta^{2 k}}{\left(1+w^{a}\right)^{\frac{1-\delta}{a}}+1} d x \\
& +C\left(p, k, \tilde{U}_{0}, \varepsilon\right)\left(1+\int_{\Omega}\left(w^{p-1+a+\delta}+w^{p+\delta}\right) \eta^{2 k-4} d x\right) .
\end{aligned}
$$

Finally, since $a<2$ we can estimate the last term by using the Young inequality as

$$
\begin{aligned}
& C\left(p, k, \tilde{U}_{0}, \varepsilon\right) \int_{\Omega}\left(w^{p-1+a+\delta}+w^{p+\delta}\right) \eta^{2 k-4} d x \\
& =C\left(p, k, \tilde{U}_{0}, \varepsilon\right) \int_{\Omega}\left(w^{1+p+\delta} \eta^{2 k-2}\right)^{\frac{p-1+a+\delta}{1+p+\delta}} \eta^{\frac{(2 k-2)(2-a)}{1+p+\delta}-2} d x \\
& \left.\quad+C\left(p, k, \tilde{U}_{0}, \varepsilon\right) \int_{\Omega}\left(w^{1+p+\delta} \eta^{2 k-2}\right)^{\frac{p+\delta}{p+1+\delta}}\right) \eta^{\frac{2 k-2}{p+1+\delta}-2} d x \\
& \leq \frac{1}{2} \int_{\Omega} w^{1+p+\delta} \eta^{2 k-2} d x \\
& \quad+C\left(p, k, \tilde{U}_{0}, \varepsilon, a\right) \int_{\Omega} \eta^{2 k-2-\frac{2(p+1+\delta)}{2-a}}+\eta^{2 k-2-2(p+1+\delta)} d x
\end{aligned}
$$

Thus, setting finally $k$ sufficiently large that

$$
k \geq 1+\frac{2(p+1+\delta)}{2-a}
$$

we see that the last term in (49) is bounded and the first term on the left hand side can be absorbed into the right hand side of (48), which then results in the following inequality

$$
\int_{\Omega} w^{1+p+\delta} \eta^{2 k-2} d x \leq \varepsilon \int_{\Omega} \frac{\left|\nabla^{2} U\right|^{2} w^{p} \eta^{2 k}}{\left(1+w^{a}\right)^{\frac{1-\delta}{a}+1}} d x+C\left(p, k, \tilde{U}_{0}, \varepsilon, a\right) .
$$

Hence, on inserting this into (45), we deduce the uniform estimate

$$
\int_{\Omega} \frac{\left|\nabla^{2} U\right|^{2} w^{p} \eta^{2 k}}{\left(1+w^{a}\right)^{\frac{1-\delta}{a}}+1} d x \leq C\left(a, \eta, k, p, \tilde{U}_{0}\right) .
$$

This however directly implies by using the uniform bound (33) that for all $\Omega_{0} \subset$ $\overline{\Omega_{0}} \subset \Omega$ and all $q \in(1, \infty)$, we have

$$
\int_{\Omega_{0}}|\nabla U|^{q} d x \leq C\left(q, a, \Omega_{0}, \tilde{U}_{0}\right) .
$$

In addition, setting now $f \equiv 1$ in (43) we see by using (52) that

$$
\int_{\Omega} \frac{\left|\nabla^{2} U\right|^{2} \eta^{2 k}}{\left(1+w^{a}\right)^{\frac{1-\delta}{a}+1}} d x \leq C(\eta) \Longrightarrow \int_{\Omega_{0}} \frac{\left|\nabla^{2} U\right|^{2}}{\left(1+w^{a}\right)^{\frac{1-\delta}{a}+1}} d x \leq C\left(\Omega_{0}\right)
$$


for all $\Omega_{0} \subset \overline{\Omega_{0}} \subset \Omega$. Consequently, combining (52) and (53) we can deduce by using the Hölder inequality that for all $q \in[1,2)$, we have

$$
\begin{aligned}
\int_{\Omega_{0}}\left|\nabla^{2} U\right|^{q} d x & =\int_{\Omega_{0}}\left(\frac{\left|\nabla^{2} U\right|^{2}}{\left(1+w^{a}\right)^{\frac{1-\delta}{a}+1}}\right)^{\frac{q}{2}}\left(1+w^{a}\right)^{\frac{q(1-\delta+a)}{2 a}} d x \\
& \leq\left(\int_{\Omega_{0}} \frac{\left|\nabla^{2} U\right|^{2}}{\left(1+w^{a}\right)^{\frac{1-\delta}{a}+1}} d x\right)^{\frac{q}{2}}\left(\int_{\Omega_{0}}\left(1+w^{a}\right)^{\frac{q(1-\delta+a)}{a(2-q)}} d x\right)^{\frac{2-q}{2}} \\
& \leq C\left(q, \Omega_{0}\right) .
\end{aligned}
$$

Let us remark that we could also add $L^{\infty}$ estimates for $\nabla U$ and consequently we could establish $\mathcal{C}^{1, \alpha}$ regularity for $U$ in the interior of $\Omega$. We omit the proof of such results here in this study since they are not essential for proving the main result.

4.2. Estimates near the boundary, where $L^{\infty}$ bound for gradients are available. In this subsection we provide the estimates similar to the preceding subsection but we focus on the estimates near that parts of the boundary $\partial \Omega$, where the $L^{\infty}$ bound for $\nabla U$ is available. Thus, for an arbitrary smooth $\Gamma \subset \partial \Omega$ assume that

$$
M:=M(\Gamma, U):=\sup _{x \in \Gamma}|\nabla U(x)|<\infty .
$$

Next, we follow almost step by step the procedure developed in the preceding subsection. Thus, we multiply (42) by $\eta^{2 k}$, where $\eta \in \mathcal{D}\left(\mathbb{R}^{2}\right)$ is nonnegative and supp $\eta \cap \partial \Omega \subset \Gamma$, and integrate twice by parts to obtain the inequality similar to (44) but we need to keep all boundary integrals. Thus, doing so, we get

$$
\begin{aligned}
(1-\delta) & \int_{\Omega} \frac{\left|\nabla^{2} U\right|^{2} f(w) \eta^{2 k}}{\left(1+w^{a}\right)^{\frac{1-\delta}{a}+1}} d x \leq C(\eta, k)\left(1+\int_{\Omega} w^{1+\delta} F_{1}(w) \eta^{2 k-2} d x\right) \\
& +\int_{\partial \Omega}\left(D_{i} F_{2}(w)-(1-\delta) D_{j}\left(\frac{D_{i} U D_{j} U}{\left(1+w^{a}\right)^{\frac{1-\delta}{a}}} F_{1}(w)\right)\right) \eta^{2 k} \boldsymbol{n}_{i} d S \\
& -\int_{\partial \Omega} F_{2}(w) \nabla \eta^{2 k} \cdot \boldsymbol{n}-(1-\delta)\left(\frac{\nabla U \otimes \nabla U}{\left(1+w^{a}\right)^{\frac{1-\delta}{a}}} F_{1}(w)\right) \cdot\left(\nabla \eta^{2 k} \otimes \boldsymbol{n}\right) d S
\end{aligned}
$$

Next, to avoid the presence of boundary integrals, we need to choose $f$ properly. Hence assuming that

$$
f(w)=0 \text { for all } w \in[0, M]
$$

we observe from (40) and (41) that

$$
F_{1}(w)=F_{1}^{\prime}(w)=F_{2}(w)=F_{2}^{\prime}(w)=0 \text { for all } w \in[0, M]
$$

and it follows from the definition of $M$ that all boundary integrals in (56) vanish provided that $f$ satisfies (57). Hence, in what follows we assume that $\delta \leq \frac{1}{2}$ and for arbitrary $p>0$ we set

$$
f(w):=\left((w-M)_{+}\right)^{p} .
$$


Thus, denoting $\Omega_{M}:=\{x \in \Omega ; w(x)>M\}$ we obtain from (56) by using similar arguments as in preceding subsection (compare with discussion above (45)) that

$$
\int_{\Omega_{M}} \frac{\left|\nabla^{2} U\right|^{2}(w-M)^{p} \eta^{2 k}}{\left(1+w^{a}\right)^{\frac{1-\delta}{a}+1}} d x \leq C(\eta, k)\left(1+\int_{\Omega_{M}} w^{1+\delta+p} \eta^{2 k-2} d x\right) .
$$

Consequently, by simple algebraic manipulations, we deduce that

$$
\int_{\Omega_{M}} \frac{\left|\nabla^{2} U\right|^{2}(w-M)^{p} \eta^{2 k}}{\left(1+(w-M)^{a}\right)^{\frac{1-\delta}{a}+1}} d x \leq C(\eta, k, M)\left(1+\int_{\Omega_{M}}(w-M)^{1+\delta+p} \eta^{2 k-2} d x\right) .
$$

Now we are exactly in the same situation as in (45) and just by replacing $w$ by $w-M$ we can repeat the same procedure to finally obtain for any $q \in(1, \infty)$

$$
\int_{\Omega_{0}}|\nabla U|^{q} d x \leq C\left(q, a, \Omega_{0}, \tilde{U}_{0}, M\right)
$$

for all $\Omega_{0} \subset \Omega$ fulfilling $\partial \Omega \cap \partial \Omega_{0} \subset \Gamma$.

Let us remark that at this point we could also provide $W^{2,2}$ estimates up to the boundary, but then the procedure (based on difference quotient techniques) is different from the one just developed. Since this is not needed for proving the main theorem we skip the proof here.

Next we focus on the estimate for $M$ on some parts of the boundary. We will first consider the domain drawn in Fig. 2. Such domains have relevance to problems in fracture mechanics and the analysis for such domains is very explicit. Then we will consider more general V-notch domains, as those sketched in Fig. 1 and characterized by the assumption (22).

Considering first the situation sketched in Fig. 2, we assume that the function $g$, occuring in (13), is nontrivial but constant only on $\Gamma_{3}$ and on $\Gamma_{5}$. Consequently, $U_{0}$ introduced in (19) is constant on $\Gamma_{i}, i=1,2,4,6,7$ and linear on $\Gamma_{3}$ and $\Gamma_{5}$. For such prescribed linear data, we will be able to show the boundedness of $\nabla U$ on $\Gamma_{2}, \ldots, \Gamma_{6}$. To do so, we briefly recall the methods of barrier functions, applicable

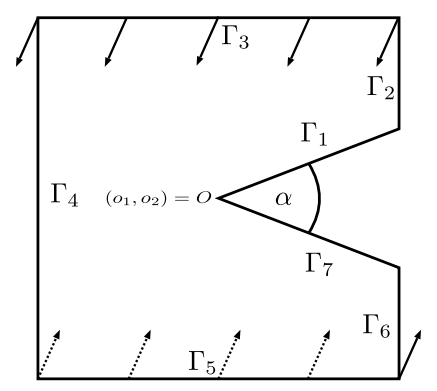

Figure 2. Geometry of the V-notch domain.

also here. Thus, for arbitrary $x_{0} \in \partial \Omega$, we say that $V_{ \pm}$is a upper/lower barrier to $U$ at $x_{0}$ if $V_{ \pm}\left(x_{0}\right)=U\left(x_{0}\right)$ and $V_{+} \geq U$ and $V_{-} \leq U$ on $\partial \Omega$. Moreover, if the upper barrier $V_{+}$is chosen such that $V_{+}$is a super-solution to (30), i.e., it fulfills

$$
\int_{\Omega} \frac{\nabla V_{+} \cdot \nabla \phi}{\left(1+\left|\nabla V_{+}\right|^{a}\right)^{\frac{1-\delta}{a}}} d x \geq 0 \text { for all non-negative } \phi \in W_{0}^{1,1+\delta}(\Omega),
$$


we can set $\phi:=\left(U-V_{+}\right)_{+}$and with the help of (30) we get

$$
\int_{\Omega}\left(\frac{\nabla U}{\left(1+|\nabla U|^{a}\right)^{\frac{1-\delta}{a}}}-\frac{\nabla V_{+}}{\left(1+\left|\nabla V_{+}\right|^{a}\right)^{\frac{1-\delta}{a}}}\right) \cdot \nabla\left(U-V_{+}\right)_{+} d x \leq 0,
$$

which due to monotonicity directly implies that

$$
U \leq V_{+} \quad \text { in } \Omega \text {. }
$$

Similarly, if $V_{-}$is a sub-solution to (30), i.e., it satisfies

$$
\int_{\Omega} \frac{\nabla V_{-} \cdot \nabla \phi}{\left(1+\left|\nabla V_{-}\right|^{a}\right)^{\frac{1-\delta}{a}}} d x \leq 0 \text { for all non-negative } \phi \in W_{0}^{1,1+\delta}(\Omega),
$$

we can conclude

$$
U \geq V_{-} \quad \text { in } \Omega .
$$

Thus, assuming that for the fixed point $x_{0} \in \partial \Omega$ we are able to construct upper and lower barriers satisfying (61) and (63) we can deduce that for all sufficiently small $h$ :

$$
\frac{V_{+}(\cdot)-V_{+}(\cdot-h \boldsymbol{n}(\cdot))}{h} \leq \frac{U(\cdot)-U(\cdot-h \boldsymbol{n}(\cdot))}{h} \leq \frac{V_{-}(\cdot)-V_{-}(\cdot-h \boldsymbol{n}(\cdot))}{h},
$$

where $\cdot$ stands for $x_{0}$. Consequently, letting $h \rightarrow 0_{+}$in the above inequalities we observe that

$$
\left|\frac{\partial U\left(x_{0}\right)}{\partial \boldsymbol{n}}\right| \leq \max _{V \pm}\left|\frac{\partial V_{ \pm}\left(x_{0}\right)}{\partial \boldsymbol{n}}\right| .
$$

Consequently, since $U=\tilde{U}_{0}$ on $\partial \Omega$ we directly have the final estimate

$$
\left|\nabla U\left(x_{0}\right)\right| \leq\left|\nabla \tilde{U}_{0}\left(x_{0}\right)\right|+\left|\nabla V_{ \pm}\left(x_{0}\right)\right| \leq\left\|\nabla \tilde{U}_{0}\right\|_{\infty}+\left|\nabla V_{ \pm}\left(x_{0}\right)\right| .
$$

The rest of this subsection, is devoted to finding proper upper and lower barriers to $U$ at "good" parts of the boundary $\partial \Omega$. Hence, our prototype barrier functions $V_{ \pm}$will always be linear functions. Then they are automatically super- and subsolutions to (30). Hence, we must check that we can always construct the linear function that is a barrier. Since we assume that at the boundary $U$ is linear it is always possible for $\Gamma_{3}, \Gamma_{4}, \Gamma_{5}$. For simplicity, we show it just for $\Gamma_{5} \subset\left\{\left(x_{1}, x_{2}\right) \in\right.$ $\left.\mathbb{R}^{2} ; x_{2}=m\right\}$ for some $m$. Then we can define our barrier functions as

$$
V_{ \pm}\left(x_{1}, x_{2}\right):=U\left(x_{1}, m\right) \pm k\left(x_{2}-m\right) .
$$

Then it is evident that $V_{ \pm}$are linear and equal to $U$ on $\Gamma_{5}$. Moreover, since $\tilde{U}_{0}$ is Lipschitz, we can always choose $k$ so large such that $V_{ \pm}$are always larger/smaller than $U$ on $\partial \Omega$. Consequently, we obtain for all $x \in \Gamma_{5}$ that

$$
\left|\nabla V_{ \pm}(x)\right| \leq\left|\nabla \tilde{U}_{0}\right|+k \leq C\left(\tilde{U}_{0}\right) .
$$

Hence, it follows from (66) that

$$
\sup _{x \in \Gamma_{5}}|\nabla U(x)| \leq \tilde{C}\left(\tilde{U}_{0}\right) .
$$

In addition, due to the special geometry and special choice of boundary conditions, we can construct barriers also on $\Gamma_{2}$ and $\Gamma_{6}$. Indeed, since $U \equiv 0$ on $\Gamma_{2} \cup \Gamma_{6}$ we can set

$$
V_{ \pm}:=0 \mp k\left(x_{1}-m\right),
$$

where $m$ is defined through $\Gamma_{2} \cup \Gamma_{6} \subset\left\{\left(x_{1}, x_{2}\right) \in \mathbb{R}^{2} ; x_{1}=m\right\}$ and $k$ is again sufficiently large. Then it is easy to check that such $V_{ \pm}$are admissible barriers. 
Next, we consider more general V-notch geometry, namely that characterized by the assumption (22) and sketched in Figure 1. We again construct the barriers provided that ${ }^{7} U_{0} \in \mathcal{C}^{1,1}$ in a neighborhood of the desired part of the boundary. For simplicity we illustrate the argument at one such point. Thus, assume that $\Omega \subset\left\{\left(x_{1}, x_{2}\right) ; x_{2}>0\right\}$ such that $(0,0) \in \partial \Omega$ and $(1,0)$ being the tangent vector. In addition, we require that $\bar{\Omega} \cap\left\{\left(x_{1}, x_{2}\right) ; x_{2}=0\right\}=(0,0)$. Finally, we assume that in a neighborhood of $(0,0) \Omega$ is a uniformly $\mathcal{C}^{1,1}$ domain. Note that it directly implies the existence of $\alpha, \beta>0$ and a $\mathcal{C}^{1,1}$ function $a:(-\alpha, \alpha) \rightarrow \mathbb{R}$ such that

$$
\begin{aligned}
& a(0)=a^{\prime}(0)=0, \quad a^{\prime \prime}\left(x_{1}\right) \geq \varepsilon \text { for almost all } x_{1} \in(-\alpha, \alpha), \\
& \Gamma_{c}:=\partial \Omega \cap\{(-\alpha, \alpha) \times(-\beta, \beta)\}=\left\{\left(x_{1}, x_{2}\right) ; x_{2}=a\left(x_{1}\right)\right\} .
\end{aligned}
$$

Then we define $V_{ \pm}$in a similar way to $(67)$. Indeed, we set

$$
V_{ \pm}\left(x_{1}, x_{2}\right):=U_{0}(0,0)+Q x_{1} \pm k x_{2}, \quad Q:=\frac{\partial U_{0}(0,0)}{\partial x_{1}}
$$

with $k$ sufficiently large. Since $V_{ \pm}$are linear functions they are automatically solutions to (30). Thus, it remains to check if they are indeed barriers. First, note that it is evident that $V_{ \pm}(0,0)=U_{0}(0,0)$. Second, we see that by choosing sufficiently large $k$ we obtain that $V_{-} \leq U \leq V_{+}$on $\partial \Omega \backslash \Gamma_{c}$. Thus, it remains to discuss only the behavior on $\Gamma_{c}$. For simplicity, we restrict ourselves to the behavior of $V_{+}$. Then for any $\left(x_{1}, x_{2}\right) \in \Gamma_{c}$ we have

$$
\begin{aligned}
& V_{+}\left(x_{1}, x_{2}\right)-U_{0}\left(x_{1}, x_{2}\right)=V_{+}\left(x_{1}, a\left(x_{1}\right)\right)-U_{0}\left(x_{1}, a\left(x_{1}\right)\right) \\
& \quad=Q x_{1}+k a\left(x_{1}\right)+U_{0}(0,0)-U_{0}\left(x_{1}, a\left(x_{1}\right)\right) \\
& \quad=k a\left(x_{1}\right)+\left(U(0,0)+\frac{\partial U_{0}(0,0)}{\partial x_{1}} x_{1}-U_{0}\left(x_{1}, 0\right)\right)+\left(U_{0}\left(x_{1}, 0\right)-U_{0}\left(x_{1}, a\left(x_{1}\right)\right)\right. \\
& \quad \geq k \varepsilon x_{1}^{2}-C\left\|\nabla^{2} U_{0}\right\|_{\infty} x_{1}^{2}-C\left\|\nabla U_{0}\right\|_{\infty} x_{1}^{2} .
\end{aligned}
$$

Consequently, we see that choosing $k$ sufficiently large we get that $V_{+} \geq U$ on $\Gamma_{c}$, which finishes this part of the proof.

However, on $\Gamma_{1}$ and $\Gamma_{7}$ we cannot use the procedure described above. We shall show in the next subsection how to get the estimates near such nonconvex parts of the boundary.

4.3. Estimates near flat parts of boundary with constant data. This part is devoted to the estimates near $\Gamma_{1}$ and $\Gamma_{7}$. To simplify the situation we rotate and shrink the domain and consider $\Gamma_{1}:=\left\{\left(x_{1}, x_{2}\right) \in \mathbb{R}^{2} ; x_{2}=0 ; x_{1} \in(0,1)\right\}$ and that for any $\varepsilon>0$ there is $\alpha>0$ such that

$$
\begin{aligned}
& \Omega_{\varepsilon}^{+}:=\left\{\left(x_{1}, x_{2}\right) ; x_{1} \in(\varepsilon, 1-\varepsilon), x_{2} \in(0, \alpha)\right\} \subset \Omega \\
& \Omega_{\varepsilon}^{-}:=\left\{\left(x_{1}, x_{2}\right) ; x_{1} \in(\varepsilon, 1-\varepsilon), x_{2} \in(-\alpha, 0)\right\} \subset \mathbb{R}^{2} \backslash \bar{\Omega} .
\end{aligned}
$$

We also set

$$
\Omega_{\varepsilon}:=\Omega_{\varepsilon}^{-} \cup \Omega_{\varepsilon}^{+} \cup\left\{\left(x_{1}, x_{2}\right) ; x_{1} \in(\varepsilon, 1-\varepsilon), x_{2}=0\right\} .
$$

\footnotetext{
${ }^{7}$ Note that in case of minimal surface equation, one does not need to assume the uniform convexity, due to the ability of finding proper barriers. However, in the setting studied in this paper we do not know yet, in particular for $a<1$, whether such barriers exist.
} 
Since $U$ is constant on $\Gamma_{1}$ we can without loss of generality assume that $U \equiv 0$ on $\Gamma_{1}$ and we can extend it as follows

$$
U\left(x_{1}, x_{2}\right):=-U\left(x_{1},-x_{2}\right) \quad \text { for any } x \in \Omega_{\varepsilon}^{-} .
$$

Note that since $U$ is zero on $\Gamma_{1}$ such an extension belongs to $W^{1,1+\delta}\left(\Omega_{\varepsilon}\right)$, in addition we keep the estimates (33) and (34), in particular we know that

$$
\|U\|_{W^{1,1+\delta}\left(\Omega_{\varepsilon}\right)}+\|U\|_{L^{\infty}\left(\Omega_{\varepsilon}\right)} \leq C .
$$

Next, for arbitrary $x \in \Omega_{\varepsilon}^{-}$we can conclude, using (26), that

$$
\begin{aligned}
-\operatorname{div} & \left.\frac{\nabla U}{\left(1+|\nabla U|^{a}\right)^{\frac{1-\delta}{a}}}\right|_{\left(x_{1}, x_{2}\right)} \\
& =\left.\frac{-\triangle U}{\left(1+|\nabla U|^{a}\right)^{\frac{1-\delta}{a}}}\right|_{\left(x_{1}, x_{2}\right)}+\left.\frac{1-\delta}{a} \frac{\nabla U \cdot \nabla|\nabla U|^{a}}{\left(1+|\nabla U|^{a}\right)^{\frac{1-\delta}{a}+1}}\right|_{\left(x_{1}, x_{2}\right)} \\
& =\left.\frac{\triangle U}{\left(1+|\nabla U|^{a}\right)^{\frac{1-\delta}{a}}}\right|_{\left(x_{1},-x_{2}\right)}-\left.\frac{1-\delta}{a} \frac{\nabla U \cdot \nabla|\nabla U|^{a}}{\left(1+|\nabla U|^{a}\right)^{\frac{1-\delta}{a}+1}}\right|_{\left(x_{1},-x_{2}\right)} \\
& =\left.\frac{\triangle_{x_{1},-x_{2}} U}{\left(1+|\nabla U|^{a}\right)^{\frac{1-\delta}{a}}}\right|_{\left(x_{1},-x_{2}\right)}-\left.\frac{1-\delta}{a} \frac{\nabla_{x_{1},-x_{2}} U \cdot \nabla_{x_{1},-x_{2}}\left|\nabla_{x_{1},-x_{2}} U\right|^{a}}{\left(1+\left|\nabla_{x_{1},-x_{2}} U\right|^{a}\right)^{\frac{1-\delta}{a}+1}}\right|_{\left(x_{1},-x_{2}\right)} \\
& =\left.\operatorname{div}_{x_{1},-x_{2}} \frac{\nabla_{x_{1},-x_{2}} U}{\left(1+\left|\nabla_{x_{1},-x_{2}} U\right|^{a}\right)^{\frac{1-\delta}{a}}}\right|_{\left(x_{1},-x_{2}\right)}=0 .
\end{aligned}
$$

Thus, $U$ solves the desired equation also in $\Omega_{\varepsilon}^{-}$. In order to show that $U$ solves the problem in $\Omega_{\varepsilon}$ we observe that for arbitrary $\phi \in \mathcal{D}\left(\Omega_{\varepsilon}\right)$ we have

$$
\begin{aligned}
0 & =-\int_{\Omega_{\varepsilon}} \phi \operatorname{div} \frac{\nabla U}{\left(1+|\nabla U|^{a}\right)^{\frac{1-\delta}{a}}} d x \\
& =\int_{\Omega_{\varepsilon}} \frac{\nabla U}{\left(1+|\nabla U|^{a}\right)^{\frac{1-\delta}{a}}} \cdot \nabla \phi d x-\int_{\varepsilon}^{1-\varepsilon} \phi\left[\frac{\frac{\partial U}{\partial x_{2}}}{\left(1+|\nabla U|^{a}\right)^{\frac{1-\delta}{a}}}\right]_{ \pm} d x_{1},
\end{aligned}
$$

where

$$
\left[\frac{\frac{\partial U}{\partial x_{2}}}{\left(1+|\nabla U|^{a}\right)^{\frac{1-\delta}{a}}}\right]_{ \pm}
$$

denotes the jump of the quantity inside. However, it follows from the construction of the extension of $U$ on $\Omega_{\varepsilon}$ that there are no jumps and consequently we have

$$
\int_{\Omega_{\varepsilon}} \frac{\nabla U}{\left(1+|\nabla U|^{a}\right)^{\frac{1-\delta}{a}}} \cdot \nabla \phi d x=0 \quad \text { for all } \varphi \in W_{0}^{1,1+\delta}\left(\Omega_{\varepsilon}\right) .
$$

Thus, we find ourselves in the situation studied in Subsection 4.1 and we may apply the interior result to get that

$$
\int_{\Omega_{\varepsilon}}|\nabla U|^{p} \leq C(p, \varepsilon)
$$

Note that in the same way one can proceed with $\Gamma_{7}$. 


\section{Proof of the MAIN THEOREM}

First, incorporating (34), (52) and (54) we can deduce for a subsequence that

$$
\begin{gathered}
U^{\delta} \rightarrow^{*} U \\
U^{\delta} \rightarrow U \\
\nabla U^{\delta} \rightarrow \nabla U
\end{gathered}
$$$$
\text { *-weakly in } L^{\infty}(\Omega) \text {, }
$$$$
\text { strongly in } W_{l o c}^{1, q}(\Omega) \quad \text { for all } q<\infty \text {, }
$$$$
\text { a.e. in } \Omega \text {. }
$$

Moreover, using (33) and Fatou's lemma we can conclude that

$$
\int_{\Omega}|\nabla U| d x \leq C
$$

Consequently, it is not difficult to let $\delta \rightarrow 0_{+}$in (30) and to get (24). To get also (23), we use the results from Subsections 4.2-4.3. Indeed, using the estimates there we can strengthen (74) to get

$$
U^{\delta} \rightarrow U \quad \text { strongly in } W^{1, q}\left(\Omega \backslash K_{\varepsilon}\right) \quad \text { for all } \varepsilon>0 \text { and all } q<\infty,
$$

where $K_{\varepsilon}$ denote the union of balls centered at all corners of $\Omega$ of radii $\varepsilon$. From this it basically follows that

$$
U=U_{0} \text { on } \partial \Omega \cap \partial\left(\Omega \backslash K_{\varepsilon}\right) .
$$

But since $\varepsilon$ is arbitrary and the trace operator is continuous from $W^{1,1}(\Omega)$ to $L^{1}(\partial \Omega)$, it directly implies $(23)$.

The uniqueness is a consequence of the strict monotonicity property of the elliptic operator that follows from (31) considering the case $\delta=0$.

The proof of Theorem 2.1 is complete.

\section{REFERENCES}

[1] Y. Antipov And P. Schiavone, Integro-differential equation for a finite crack in a strip with surface effects, Quart. J. Mech. Appl. Math., 64 (2011), pp. 87-106.

[2] M. F. Beatty and M. A. Hayes, Deformations of an elastic internally constrained materials. 1. homogeneous deformation, Journal of Elasticity, 29 (1992), pp. 1-84.

[3] — Deformations of an elastic internally constrained materials. 2. non-homogeneous deformation, Quarterly Journal of Applied Mathematics, 45 (1992), pp. 663-709.

[4] - Deformations of an elastic internally constrained materials. 3. small superimposed deformations and waves, Zeitschrift fur Angewandte Mathematick und Physik, 46 (1995), pp. $72-106$.

[5] J. F. BELL, Contemporary perspectives in finite strain plasticity, International Journal of Plasticity, 1 (1985), pp. 3-27.

[6] — Experiments in the kinematics of large plastic strain in ordered materials, International Journal of Solids and Structures, 25 (1989), pp. 268-278.

[7] K. B. Broberg, Cracks and fracture, Academic Press, San Diego, 1999.

[8] R. Bustamante and K. R. Rajagopal, Solutions of some simple boundary value problems within the context of a new class of elastic materials, International Journal of Non-Linear Mechanics, 46 (2011), pp. 376-386.

[9] G. P. Cherepanov, Fracture, Krieger Publishing Company, Malabar, 1998.

[10] E. De GIORGI, Sulla differenziabilità e l'analiticità delle estremali degli integrali multipli regolari, Mem. Accad. Sci. Torino. Cl. Sci. Fis. Mat. Nat. (3), 3 (1957), pp. 25-43.

[11] U. Dierkes, S. Hildebrandt, A. Küster, And O. Wohlrab, Minimal surfaces. I, vol. 295 of Grundlehren der Mathematischen Wissenschaften [Fundamental Principles of Mathematical Sciences], Springer-Verlag, Berlin, 1992. Boundary value problems.

[12] - Minimal surfaces. II, vol. 296 of Grundlehren der Mathematischen Wissenschaften [Fundamental Principles of Mathematical Sciences], Springer-Verlag, Berlin, 1992. Boundary regularity. 
[13] R. Finn, Remarks relevant to minimal surfaces, and to surfaces of prescribed mean curvature, J. Analyse Math., 14 (1965), pp. 139-160.

[14] G. Galilei, Discorsi e dimostrazioni matematiche intorno a due nuove scienze attenenti alla mecanica ed ei movimenti locali, Dover, New York, 1954. original published in 1638. Engl. transl. "Dialogue concerning two new sciences".

[15] E. Giusti, Minimal surfaces and functions of bounded variation, vol. 80 of Monographs in Mathematics, Birkhäuser Verlag, Basel, 1984.

[16] M. E. Gurtin and A. I. Murdoch, A continuum theory of elastic material surfaces, Arch. Rat. Mech. Anal., 57 (1975), pp. 291-323.

[17] M. F. Kaninnen And C. H. Popelar, Advanced fracture mechanics, Oxford University Press, New York, 1985.

[18] C. Kim, P. Schiavone, and C.-Q. Ru, Analysis of a mode-iii crack in the presence of surface elasticity and a prescribed non-uniform surface traction, Zeitschrift fur Angewandte Mathematik und Physik, 61 (2010), pp. 555-564.

[19] - The effects of surface elasticity on an elastic solid with mode-III crack: Complete solution, Journal of Applied Mechanics, 77 (2010).

$[20]$ - Analysis of plane-strain crack problems (mode-i and mode-ii) in the presence of surface elasticity, Journal of Elasticity, 104 (2011), pp. 397-420.

[21] — Effect of surface elasticity on an interface crack in plane deformations, Proc. Roy. Soc. A, 467 (2011), pp. 3530-3549. doi:10.1098/rspa.2011.0311.

[22] J. K. Knowles, The finite anti-plane shear field near the tip of the crack for a class of incompressible elastic solids, Int. J. Fract., 13 (1997), pp. 611-639.

[23] V. Kulvait, J. Málek, And K. Rajagopal, Anti-plane stress state of a plate with a V-notch for a new class of elastic solids, Int. J. Fract., (2012), pp. 1-15. doi:10.1007/s10704-012-97725 .

[24] G. M. Lieberman, Boundary regularity for solutions of degenerate elliptic equations, Nonlinear Anal., 12 (1988), pp. 1203-1219.

[25] A. NADAI, Theory of flow and fracture of solids, McGraw-Hill, New York, 1950.

[26] J. NAsh, Continuity of solutions of parabolic and elliptic equations, Amer. J. Math., 80 (1958), pp. 931-954.

[27] J. NEČAS, Sur la régularité des solutions variationelles des équations elliptiques non-linéaires d'ordre $2 k$ en deux dimensions, Ann. Scuola Norm. Sup. Pisa (3), 21 (1967), pp. 427-457.

[28] J. NEČAS, Sur la régularité des solutions faibles des équations elliptiques non linéaires, Comment. Math. Univ. Carolinae, 9 (1968), pp. 365-413.

[29] J. C. C. Nitsche, On the non-solvability of Dirichlet's problem for the minimal surface equation, J. Math. Mech., 14 (1965), pp. 779-788.

[30] E. S. Oh, J. R. Walton, and J. C. Slattery, A theory of fracture based upon an extension of continuum mechanics to the nanoscale, Journal of Applied Mechanics, 73 (2006), pp. 792798.

[31] K. R. Rajagopal, On implicit constitutive theories, Appl. Math., 48 (2003), pp. 279-319.

[32] — Elasticity of elasticity, Zeitschrift fur Angewandte Math Phys, 58 (2007), pp. 309-417.

[33] — Conspectus of concepts of elasticity, Math. Mech. Solids, 16 (2011), pp. 536-562.

[34] — Non-linear elastic bodies exhibiting limiting small strain, Math. Mech. Solids, 16 (2011), pp. 122-139.

[35] - On a new class of models in elasticity, J. Math. Comp. Appl., 15 (2011), pp. 506-528.

[36] K. R. Rajagopal and A. R. SRinivasa, On the response of non-dissipative solids, Proc. Roy. Soc. London A, 463 (2007), pp. 357-367.

[37] — On a class of non-dissipative materials that are not hyperelastic, Proc. Roy. Soc. London A, 465 (2009), pp. 493-500.

[38] K. R. Rajagopal and L. TaO, On the response of non-dissipative solids, Commun. Nonlinear Sci. Numer. Simul., 13 (2008), pp. 1089-1100.

[39] K. R. Rajagopal and J. Walton, Modeling fracture in the context of strain-limiting theory of elasticity, Int. J. Fract., 169 (2011), pp. 39-48.

[40] T. Sendova And J. R. Walton, A new approach to the modeling and analysis of fracture through extension of continuum mechanics to the nanoscale, Math Mech Solids, 15 (2010), pp. 368-413.

[41] J. C. Slattery, L. Sagis, And E.-S. Oh, Interfacial Transport Phenomena, Springer, Berlin, 2007. 
[42] V. SouČEK, The nonexistence of a weak solution of Dirichlet's problem for the functional of minimal surface on nonconvex domains, Comment. Math. Univ. Carolinae, 12 (1971), pp. $723-736$.

[43] J. StaRÁ, Regularity results for non-linear elliptic systems in two dimensions, Ann. Scuola Norm. Sup. Pisa (3), 25 (1971), pp. 163-190.

[44] E. Sternberg and J. K. Knowles, Finite-deformation analysis of elastostatic field near tip of a crack-reconsideration and higher order results, Journal of Elasticity, 4 (1974), pp. 201233.

[45] E. Sternberg And J. K. Knowles, Failure of ellipticity and emergence of discontinuous deformations gradients in plane finite elastostatics, Journal of Elasticity, 8 (1978), pp. 329379 .

[46] E. Sternberg and J. K. Knowles, Discontinuous deformation gradients near the tip of a crack in finite anti-plane shear-example, Journal of Elasticity, 10 (1980), pp. 81-110.

[47] E. Sternberg and J. K. Knowles, Anti-plane shear fields with discontinueous deformation gradients near the tip of a crack in finite elastostatics, Journal of Elasticity, 11 (1981), pp. 129-164.

[48] A. M. TARAntino, Nonlinear fracture mechanics for an elastic Bell material, Quart. J. Mech. Appl. Math., 50 (1997), pp. 436-456.

[49] J. Walton, A note on fracture models incorporating surface elasticity, Journal of Elasticity, (2011), pp. 1-8. 10.1007/s10659-011-9369-7.

Charles University in Prague, Faculty of Mathematics and Physics, Mathematical Institute, Sokolovská 83, 18675 Prague 8, Czech Republic

E-mail address: mbul8060@karlin.mff.cuni.cz

Charles University in Prague, Faculty of Mathematics and Physics, Mathematical Institute, Sokolovská 83, 18675 Prague 8, Czech Republic

E-mail address: malek@karlin.mff.cuni.cz

Department of Mechanical Engineering, Texas A\&M University, College Station, TX 77843 , USA

E-mail address: krajagopal@tamu.edu

Department of Mathematics, Texas A\&M University, College Station, TX 77843, USA

E-mail address: jwalton@tamu.edu 